\title{
The structure and role of lactone intermediates in linkage-specific sialic acid derivatization reactions
}

\author{
$\operatorname{Tamas}$ Pongracz ${ }^{1}$ (D) $\cdot$ Aswin Verhoeven ${ }^{1}$ (i) $\cdot$ Manfred Wuhrer $^{1}$ (i) $\cdot$ Noortje de Haan ${ }^{1,2}$ (i)
}

Received: 22 September 2020 / Revised: 21 December 2020 / Accepted: 30 December 2020 / Published online: 18 January 2021

(C) The Author(s) 2021

\begin{abstract}
Sialic acids occur ubiquitously throughout vertebrate glycomes and often endcap glycans in either $\alpha 2,3$ - or $\alpha 2,6$-linkage with diverse biological roles. Linkage-specific sialic acid characterization is increasingly performed by mass spectrometry, aided by differential sialic acid derivatization to discriminate between linkage isomers. Typically, during the first step of such derivatization reactions, in the presence of a carboxyl group activator and a catalyst, $\alpha 2,3$-linked sialic acids condense with the subterminal monosaccharides to form lactones, while $\alpha 2,6$-linked sialic acids form amide or ester derivatives. In a second step, the lactones are converted into amide derivatives. Notably, the structure and role of the lactone intermediates in the reported reactions remained ambiguous, leaving it unclear to which extent the amidation of $\alpha 2,3$-linked sialic acids depended on direct aminolysis of the lactone, rather than lactone hydrolysis and subsequent amidation. In this report, we used mass spectrometry to unravel the role of the lactone intermediate in the amidation of $\alpha 2,3$-linked sialic acids by applying controlled reaction conditions on simple and complex glycan standards. The results unambiguously show that in common sialic acid derivatization protocols prior lactone formation is a prerequisite for the efficient, linkage-specific amidation of $\alpha 2,3$-linked sialic acids, which proceeds predominantly via direct aminolysis. Furthermore, nuclear magnetic resonance spectroscopy confirmed that exclusively the $\mathrm{C} 2$ lactone intermediate is formed on a sialyllactose standard. These insights allow a more rationalized method development for linkage-specific sialic derivatization in the future.
\end{abstract}

Keywords Sialic acid $\cdot$ Linkage isomers $\cdot$ Lactone $\cdot$ Mass spectrometry $\cdot$ Nuclear magnetic resonance $\cdot$ Glycomics

\section{Introduction}

Protein glycosylation is a ubiquitous co- and post-translational modification, which has lately received considerable attention given its relevance in a multitude of biological processes [1, 2]. Glycosylation affects folding and solubility of glycoproteins, and changes in response to diverse environmental cues [3, 4]. Sialic acids are monosaccharides which are endcapping glycans, where they play important roles in either masking the glycoprotein from its surroundings or by mediating interaction with glycan-binding proteins [5]. The importance of sialylated glycans is exemplified by their broad

Noortje de Haan

n.de_haan@lumc.nl

1 Center for Proteomics and Metabolomics, Leiden University Medical Center, 2333ZA Leiden, The Netherlands

2 Present address: Copenhagen Center for Glycomics, University of Copenhagen, 2200 Copenhagen, Denmark involvement in host-pathogen interactions [6-8], glycoprotein half-life in the circulation [9], lymphocyte homing [10], inflammation [11] and tumor development $[12,13]$. Of note, sialic acids typically terminate glycans in $\alpha 2,3-$ or $\alpha 2,6$-linkages, adding an additional layer of functional complexity to the glycan moiety [5].

To assess sialic acid linkages qualitatively and quantitatively, an array of techniques has been developed relying on the differential derivatization of the carboxyl groups of the isomers and their detection by mass spectrometry (MS) $[6,14-20]$. In the presence of a carboxylic acid activator (such as 1-ethyl-3-(3-dimethylaminopropyl) carbodiimide; EDC [21]) and a catalyst (such as 1-hydroxybenzotriazole; HOBt [22]) $\alpha 2,6$-linked sialic acids react with added amines or alcohols to form amide or ester derivatives, respectively. In contrast, $\alpha 2,3$-linked sialic acids form lactones under the same conditions (Fig. 1) [14, 20]. Because of the limited stability of the lactones, a second reaction step is often included to convert them into amide products $[15,23]$. 


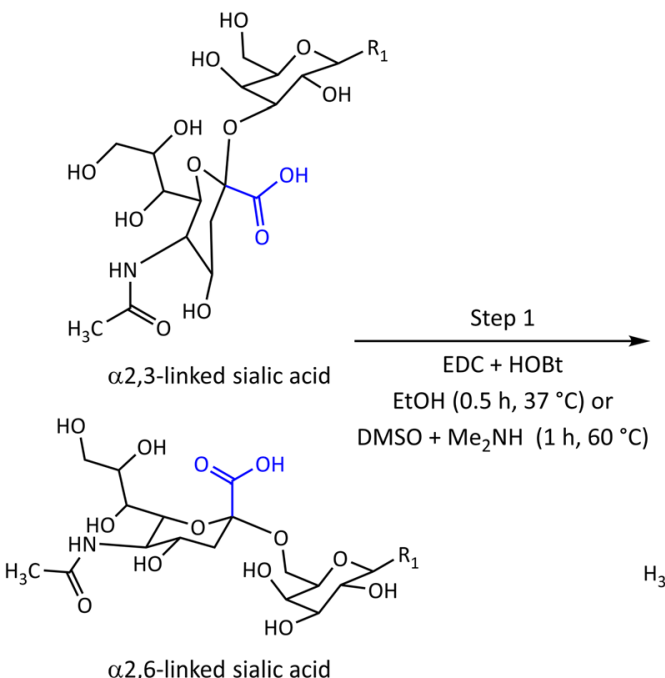

$\alpha 2,6$-linked sialic acid

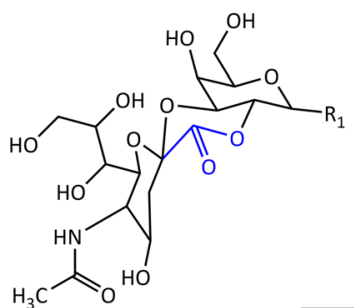

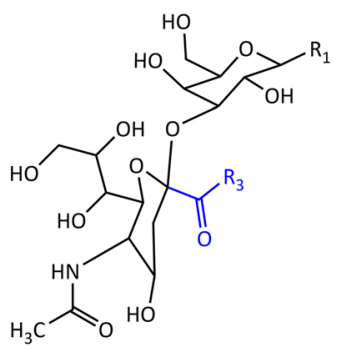

$\mathrm{EDC}+\mathrm{HOB}$

$\mathrm{NH}_{4} \mathrm{OH}$ or $\mathrm{MeNH}_{2}$ EtOH $\left(0.5 \mathrm{~h}, 37^{\circ} \mathrm{C}\right)$ or

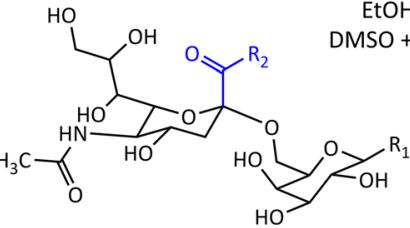

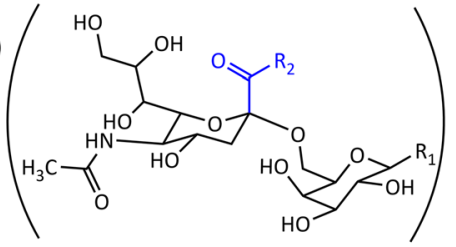

$\mathrm{R}_{1}$ : remainder of glycoconjugate $R_{2}$ : ethyl ester or dimethyamine amide

$\mathrm{R}_{3}$ : ammonia amide or methylamine amide
Fig. 1 Schematic representation of common one-pot in-solution linkagespecific derivatization approaches for sialylated glycoconjugates. In the first step, $\alpha 2,3$-linked sialic acids form a lactone with the subterminal galactose, while $\alpha 2,6$-linked sialic acids are subjected to ethyl

Four different approaches have been described to get from lactonized $\alpha 2,3$-linked sialic acids to a stable end product: 1) Lactones were purified and hydrolyzed under alkaline conditions before treating them with a second nucleophile in the presence of a carboxyl activator $[17,19]$. 2) Lactones were subjected to a mild clean-up step and amidated in a ringopening reaction in the sole presence of an amine (aminolysis) [24]. 3) The second nucleophile was directly added to the reaction mixture to obtain the stable derivative of the $\alpha 2,3$-linked sialic acids [16, 23, 25]. 4) Lactones were purified under mild conditions at neutral $\mathrm{pH}$ and treated with a second nucleophile in the presence of a carboxyl activator and catalyst [16]. While the first approach relies on the reaction of the unmodified carboxyl group and the second relies on direct aminolysis of the lactone, for approaches 3 and 4 the reaction path remains unclear. Specifically, one may speculate that direct lactone aminolysis may results in the stable derivative of $\alpha 2,3$-linked sialic acids in these cases. Alternatively, lactones may be opened by hydrolysis followed by derivatization of the free carboxyl group [26]. Research in the field of linkage-specific sialic acid derivatization has been abundant, with the methodological advances realized as of today summarized in two succinct reviews [26, 27]. However, while one-pot reactions such as approach 3 (Fig. 1) are gaining popularity due to their ease of use and versatility, the conversion of the lactone intermediate into a stable derivative - albeit a key part of the approach - is insufficiently understood, thereby hampering the further design and optimization of the approach.

In order to address this gap in understanding, we here studied the lactone dependency of the amidation step of $\alpha 2,3-$ esterification or $\mathrm{Me}_{2} \mathrm{NH}$ (dimethylamine) amidation. In the second step, the lactone undergoes mainly direct aminolysis by $\mathrm{NH}_{3}$ (ammonia) or $\mathrm{MeNH}_{2}$ (methylamine), and the $\alpha 2,6$-sialyllactose derivative remains unchanged

sialylated glycans in one-pot sialic acid derivatization approaches. For this, we used an $\alpha 2,3$-sialyllactitol standard and $\alpha 2,3$-sialylated, complex-type $N$-glycans of recombinant human erythropoietin (rhEPO). In addition, we employed NMR spectroscopy to characterize the configuration of the lactone for an $\alpha 2,3$-sialyllactose standard under representative conditions used in differential sialic acid derivatization.

\section{Materials and methods}

\section{Chemicals, reagents and enzymes}

All materials and reagents used in this study were of analytical grade and purchased from commercial suppliers. Type I Ultrapure Water was produced by an ELGA Purelab Ultra system (Elga LabWater, High Wycombe, UK) and used throughout. Ethanol (EtOH), sodium hydroxide $(\mathrm{NaOH})$, SDS (sodium dodecyl sulfate), TFA (trifluoroacetic acid) and disodium hydrogen phosphate dihydrate $\mathrm{Na}_{2} \mathrm{HPO}_{4} \cdot 2 \mathrm{H}_{2} \mathrm{O}$ ), potassium dihydrogen phosphate $\left(\mathrm{KH}_{2} \mathrm{PO}_{4}\right)$, sodium borohydride $\left(\mathrm{NaBH}_{4}\right)$, sodium chloride $(\mathrm{NaCl})$ and Dowex $50 \mathrm{~W}$ X8 cation exchange resin were purchased from Merck (Darmstadt, Germany). Glacial acetic acid and potassium hydroxide were obtained from Honeywell Fluka (Charlotte, NC). 1-Hydroxybenzotriazole (HOBt) hydrate, ortho-phosphoric acid, $40 \mathrm{wt} . \%$ aqueous methylamine $\left(\mathrm{MeNH}_{2}\right), 40 \mathrm{wt} . \%$ aqueous dimethylamine $\left(\mathrm{Me}_{2} \mathrm{NH}\right), 28$ 30 wt. \% aqueous ammonium hydroxide $\left(\mathrm{NH}_{4} \mathrm{OH}\right)$, anhydrous DMSO and a mixture of 2,5-dihydroxybenzoic acid and 2hydroxy-5-methoxybenzoic acid (super-DHB; sDHB) were 
obtained from Sigma-Aldrich (Steinheim, Germany), while 1ethyl-3-(3-dimethylaminopropyl) carbodiimide (EDC) hydrochloride was acquired from Fluorochem (Hadfield, UK). HPLC Supra-gradient acetonitrile $(\mathrm{ACN})$ originated from Biosolve BV (Valkenswaard, Netherlands), and Peptide Calibration Mix II from Bruker Daltonics (Bremen, Germany). Recombinant peptide-N-glycosidase F (PNGase F) was purchased from Roche Diagnostics (Mannheim, Germany). Acidic PBS (pH 5.6) was prepared as described previously [24]. The $N$-glycan release mixture was composed of $10 \mu \mathrm{L} 4 \%$ Nonidet P-40 supplement (VWR International, Solon, $\mathrm{OH}), 10 \mu \mathrm{L} 5 \mathrm{x}$ acidic PBS, and $1 \mathrm{U}$ PNGase F. 2,2,3,3D4 sodium trimethylsilylproprionate (TSP) was obtained from Cambridge Isotope Laboratories (Tewksburry, MA), and deuterated water $\left(\mathrm{D}_{2} \mathrm{O}\right)(99.8$ atom $\%$ D) from Cortecnet (Voisins-le-Bretonneux, France).

\section{Samples}

Commercially available sialyllactose (SL) and sialylLacNAc (SLN) standards with known sialic acid linkage $(\alpha 2,3$ or $\alpha 2,6)$ were obtained from Carbosynth (Compton, UK). SL and SLN standards were dissolved to a final concentration of $10 \mu \mathrm{g} / \mu \mathrm{L}$. 2,3-SL samples for NMR analysis were prepared at a final concentration of $5 \mathrm{mg} / \mu \mathrm{L}$ (reference NMR sample without sialic acid derivatization) or $1 \mathrm{mg} / \mu \mathrm{L}$ (with sialic acid derivatization) in $\mathrm{D}_{2} \mathrm{O}$. The NMR sample with sialic acid derivatization was prepared in triplicate, pooled and added up to $200 \mu \mathrm{L}$ final volume with $\mathrm{D}_{2} \mathrm{O}$ before analysis. Chinese hamster ovary $(\mathrm{CHO})$ cell culture-derived rhEPO was kindly provided by Roche Diagnostics (Penzberg, Germany), and immunoglobulin $\mathrm{G}$ ( $\mathrm{IgG}$ ) affinity purified from normal human plasma was obtained from Athens Research \& Technology Inc. (Athens, GA).

\section{Reduction of sialyllactose}

Sodium borohydride-reduced SL (sialyllactitol) standards were prepared according to established procedures [28], but by replacing methanol with isopropanol throughout to prevent methyl esterification of the carboxylic acids. The dried samples were reconstituted in water to a final concentration of $10 \mu \mathrm{g} / \mu \mathrm{L}$.

\section{PNGase F $\mathrm{N}$-glycan release}

$N$-glycans from rhEPO and IgG were released in acidic PBS [21]. Briefly, $20 \mu \mathrm{L} 2 \%$ SDS was added to $10 \mu \mathrm{L}$ rhEPO $(50 \mu \mathrm{g})$ or $\mathrm{IgG}(41.8 \mu \mathrm{g})$ standard and shaken for $5 \mathrm{~min}$ on a horizontal shaking platform at $1350 \mathrm{rpm}$ (rotary motion of $1.5 \mathrm{~mm}$ ) followed by $10 \mathrm{~min}$ incubation at $60^{\circ} \mathrm{C}$. The sample was allowed to come to room temperature before the addition of $20 \mu \mathrm{L}$ release mixture. The samples were shaken for $5 \mathrm{~min}$ at $1350 \mathrm{rpm}$, followed by overnight incubation at $37^{\circ} \mathrm{C}$, and stored at $-20{ }^{\circ} \mathrm{C}$ until sialic acid derivatization.

\section{Preparation of sialic acid derivatization reagents}

The ethyl esterification reagent (EE reagent) was prepared by dissolving EDC and HOBt in EtOH to a final concentration of $0.25 \mathrm{M}$ of both chemicals. The dimethylamine amidation reagent (DMA reagent) was prepared by dissolving EDC and HOBt and adding $40 \% \mathrm{Me}_{2} \mathrm{NH}$ to DMSO in a final concentration of 0.25 and 0.5 and $0.25 \mathrm{M}$, respectively. The controlreagents were $100 \% \mathrm{EtOH}$ and $0.25 \mathrm{M} \mathrm{Me}_{2} \mathrm{NH}$ in DMSO. The $\mathrm{pH}$ of all reagents was measured in triplicates using narrow range $\mathrm{pH}$ indicator strips, after ten times dilution in water (Supplementary Table 1).

\section{Linkage-specific sialic acid derivatization}

Ethyl esterification (EE) and $\mathrm{Me}_{2} \mathrm{NH}$ amidation (DMA) were performed according to established procedures [14, 20]. Briefly, $20 \mu \mathrm{L}$ EE reagent or DMA reagent was added to the wells of a 96-well NUNC V-bottom plate (Thermo Scientific, Waltham, MA). Then, the reactions were performed by adding $1 \mu \mathrm{L}$ of one of the SL standards $(10 \mu \mathrm{g})$, sialyllactitol $(10 \mu \mathrm{g})$ or released $N$-glycan mixture, followed by 30 or 60 min incubation at 37 or $60{ }^{\circ} \mathrm{C}$ for $\mathrm{EE}$ and DMA, respectively. In case of ethyl esterification $+\mathrm{NH}_{3}$ amidation $(\mathrm{EE}+$ AA) $[25,29]$, ethyl esterification $+\mathrm{MeNH}_{2}$ amidation (EE + MA), $\mathrm{Me}_{2} \mathrm{NH}+\mathrm{NH}_{3}$ amidation (DMA + AA) [23], or $\mathrm{Me}_{2} \mathrm{NH}$ amidation $+\mathrm{MeNH}_{2}$ amidation (DMA + MA), $4 \mu \mathrm{L}$ of $\mathrm{NH}_{4} \mathrm{OH}$ (final concentration of $1.34 \mathrm{M}$ ) or $\mathrm{MeNH}_{2}$ (final concentration of $1.83 \mathrm{M}$ ) were added to the reaction mixture followed by further incubation for $30 \mathrm{~min}$ (in case of EE + AA and $\mathrm{EE}+\mathrm{MA}$ ) or $120 \mathrm{~min}$ (in case of $\mathrm{DMA}+\mathrm{AA}$ and $\mathrm{DMA}+\mathrm{MA})$. The direct amidation reagents were prepared by adding $4 \mu \mathrm{L}$ of $\mathrm{NH}_{4} \mathrm{OH}$ or $\mathrm{MeNH}_{2}$ to the reagents right before sample addition. All samples were prepared in triplicate.

\section{Cotton HILIC SPE}

Cotton HILIC SPE of sialic acid-derivatized SL and released $N$-glycans was performed according to established procedures described elsewhere [22, 30]. Briefly, following derivatization, ACN was added to the reaction mixture to $50 \% \mathrm{v} / \mathrm{v}$ $(\mathrm{EE} \pm \mathrm{AA}$ or $\mathrm{MA})$ or $85 \% \mathrm{v} / \mathrm{v}(\mathrm{DMA} \pm \mathrm{AA}$ or $\mathrm{MA})$ followed by cotton-HILIC purification. The retained glycans were eluted in $10 \mu \mathrm{L}$ water. For NMR analysis, the sample was washed with and eluted into $\mathrm{D}_{2} \mathrm{O}$. To prepare the glycan samples for MALDI-TOF-MS, $1 \mu \mathrm{L}$ sDHB $(5 \mathrm{mg} / \mathrm{mL}$ in $50 \%$ ACN with $1 \mathrm{mM} \mathrm{NaOH}$ ), while for MALDI-FT-ICR-MS, $1 \mu \mathrm{L}$ sDHB ( $5 \mathrm{mg} / \mathrm{mL}$ in $50 \% \mathrm{ACN}$ with $0.1 \mathrm{mM} \mathrm{NaOH}$ ) was spotted on an AnchorChip 800/384 TF MALDI target (Bruker Daltonics) 
topped with $1 \mu \mathrm{L}$ HILIC enriched glycans. The spots were left to dry by air.

\section{MALDI-TOF-MS}

MALDI-TOF-MS analyses were performed on an UltrafleXtreme mass spectrometer equipped with a Smartbeam-II laser (Bruker Daltonics, Bremen, Germany). Spectra were acquired in reflectron positive mode collecting a total of 10,000 laser shots at a laser frequency of $1000 \mathrm{~Hz}$, using $25 \mathrm{kV}$ acceleration voltage. Prior to measurement, the instrument was calibrated with Peptide Calibration Mix II (Bruker Daltonics). An $m / z$ range of 300-1000 was used for all SL, SLN, and sialyllactitol measurements.

\section{MALDI-FT-ICR-MS}

MALDI-FT-ICR-MS analyses were performed on a $15 \mathrm{~T}$ solariX XR FT-ICR mass spectrometer equipped with a CombiSource, a ParaCell and a Smartbeam-II laser (Bruker Daltonics, Bremen, Germany). Spectra were acquired in positive mode. Prior to measurement, the instrument was calibrated using CsI cluster masses for the analysis of methyl esterified $\alpha 2,3$-sialyllactitol, or Peptide Calibration Mix II (Bruker Daltonics) for the measurement of rhEPO and IgG released $N$ glycans. For each spot, an average spectrum was obtained from the acquisition of 10 spectra in the $m / z$ range of $153-$ 1000 for sialyllactitol or 1011-5000 for released $N$-glycans, using $1 \mathrm{M}$ data points.

\section{MS data analysis}

Data analysis was performed with the in-house developed software MassyTools (version number 1.02200129a) [31]. Internal calibration was performed based on a selected calibrant list (Supplementary Table 2-4), followed by targeted data extraction using predefined glycan compositions. Data quality control was performed based on quality control parameters (isotopic pattern quality (IPQ), ppm error, and $\mathrm{S} / \mathrm{N}$ ). Total area normalization of the extracted glycan signals passing quality control criteria (IPQ $<0.2$; ppm deviation $<15 \mathrm{ppm}$; $\mathrm{S} / N>3$ ) was performed for each spectrum (Supplementary Table 5-7). Analytes resulting in overlapping signals (e.g. the $\mathrm{NH}_{3}$ amidated SL or sialyllactitol and their unmodified counterpart) were corrected based on the theoretical overlap between their isotopic patterns. Averages and SDs were calculated from triplicate measurements using Microsoft Excel.

\section{NMR spectroscopy}

All NMR data was recorded on a Bruker AVANCE II spectrometer equipped with a $14.1 \mathrm{~T}$ magnet and a $5 \mathrm{~mm}$ TCI cryogenic probe head and a z-gradient system. The samples were manually injected into disposable $5 \mathrm{~mm}$ SampleJet NMR tubes and sealed with a closed cap. A Bruker SampleJet system was used for sample insertion, removal and temporary storage. In the SampleJet the samples were kept at a temperature of $6{ }^{\circ} \mathrm{C}$. The temperature for the NMR measurements was $300 \mathrm{~K}$, which was carefully calibrated using a fresh methanol-d4 sample. TSP was used as chemical shift reference. All sample were measured in an 1D NOESY ${ }^{1} \mathrm{H}$ NMR experiment with $25 \mathrm{~Hz}$ water presaturation and a relaxation delay of $4 \mathrm{~s}$. The reference $\alpha 2,3$-sialyllactose was measured by accumulating 4 scans, for the lactone sample 256 scans were collected. The lactonized $\alpha 2,3$-sialyllactose was analyzed directly after preparation, as well as after $16 \mathrm{~h}$ of storage at $300 \mathrm{~K}$. The raw NMR data was processed and analyzed in Bruker TopSpin 3.0.

\section{Results and discussion}

Here, we present a set of experiments performed to unravel the role of the lactone intermediate in the amidation during linkage-specific sialic acid derivatization. We further present the structural characterization of the lactone intermediate formed with common linkage-specific sialic acid derivatization conditions.

\section{The role of the lactone intermediate in the amidation of sialyllactitol}

The lactone dependency of the amidation reaction was investigated using an $\alpha 2,3$-linked sialyllactitol standard. Sialyllactitol was chosen as a suitable standard as it lacked the reducing end aldehyde, thereby avoiding reducing end associated side reactions (Supplementary Fig. 1) (Supplementary Table 5). In order to avoid methyl ester side products arising during sialyllactitol preparation, the methanol used in the original protocol during cleanup [28] was replaced by isopropanol (Supplementary Fig. 2 and 3).

To resolve the ambiguity around the lactone dependency of amidation, first the lactone formation of $\alpha 2,3$-sialyllactitol was promoted based on preceding reports using the EE or DMA reagent $[22,23]$. Using both conditions, $\alpha 2,3-$ sialyllactitol underwent near complete intramolecular water loss ( $m / z$ 640.204) (Fig. 2a, d and Fig. 3) (Supplementary Table 6). In the second step, conditions were set as to promote $\mathrm{NH}_{3}$ amidation [23, 25] or $\mathrm{MeNH}_{2}$ amidation [16, 24]. High conversion efficiency was observed for the lactonized species following all conditions resulting in the ammonia amide at $\mathrm{m} / \mathrm{z}$ 657.235, and methylamide at $m / z, 671.250$ (Fig. 2 and Fig. 3) (Supplementary Table 6). While non-selective conversion of the standard was scarcely observed using EE reagent $(0.2 \pm$ $0.02 \%$ ethyl ester formation for $\alpha 2,3$-sialyllactitol; $\mathrm{m} / \mathrm{z}$ 

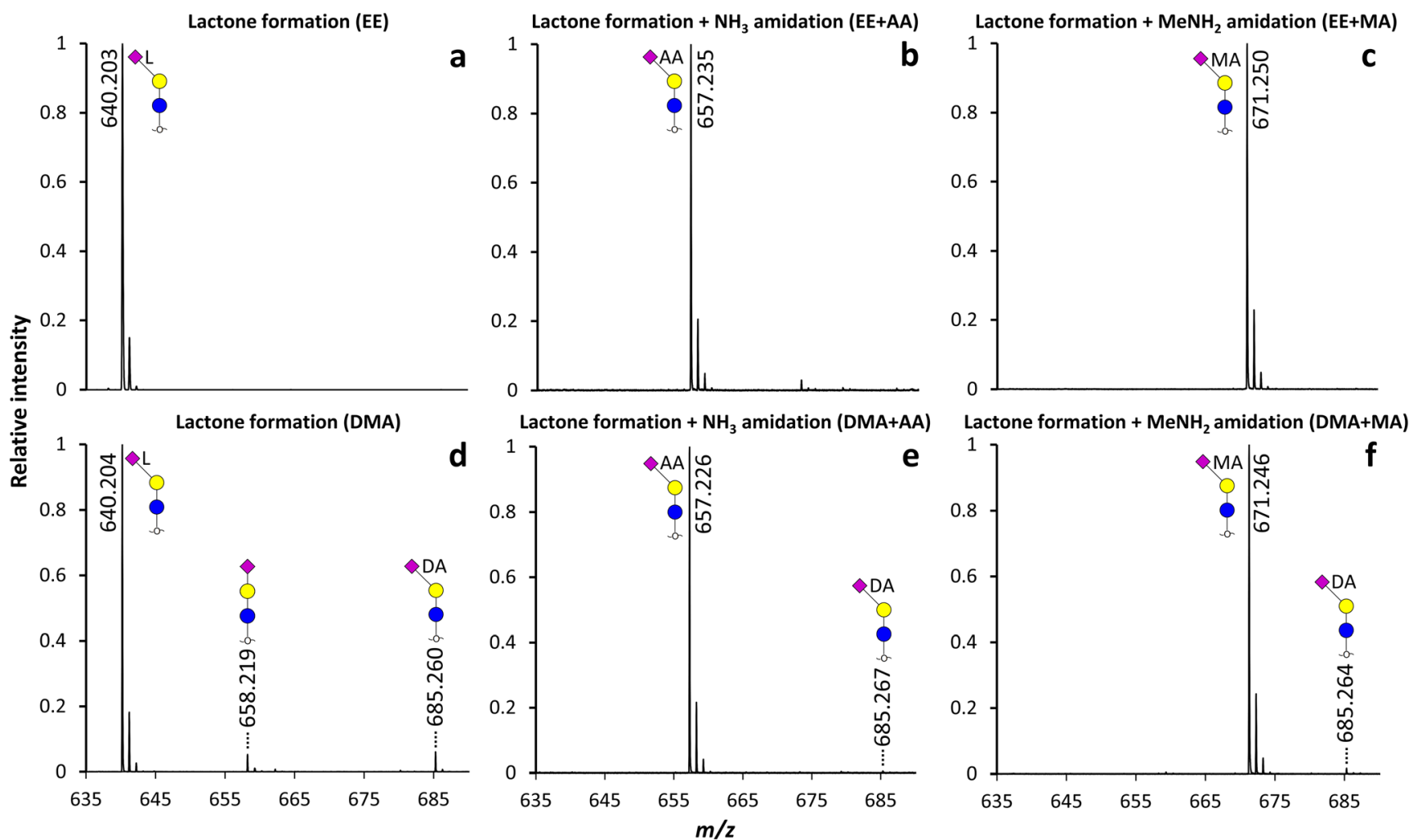

Fig. 2 Representative MALDI-TOF-MS spectra showing the modifications induced on an $\alpha 2,3$-sialyllactitol standard under native reaction conditions in EtOH (a-c) or DMSO (d-f). Symbols indicate the monosaccharide residues glucose (blue circle), galactose (yellow circle) and $\mathrm{N}$ acetylneuraminic acid (purple diamond). In case of derivatized sialic acids, an $\alpha 2,3$-linkage is depicted with a left angle, with lactonization indicated by $\mathrm{L}, \mathrm{NH}_{3}$ amidation by $\mathrm{AA}, \mathrm{MeNH}_{2}$ amidation by $\mathrm{MA}$, and $\mathrm{Me}_{2} \mathrm{NH}$ amidation by DA next to the sialic acid residue. Non-derivatized sialic acids are depicted without an angle. All species were detected as $[\mathrm{M}+\mathrm{Na}]^{+}$
686.243), in case of DMA reagent, the $\mathrm{Me}_{2} \mathrm{NH}$ amidated $\alpha 2,3$-sialyllactitol by-product showed $3.7 \pm 0.6 \%$ relative abundance ( $\mathrm{m} / \mathrm{z} 685.260$ (Fig. 2d and Fig. 3)). This can be explained by the higher nucleophilicity of $\mathrm{Me}_{2} \mathrm{NH}$ as compared to $\mathrm{EtOH}$, leading to higher rates of misconversion, yet the linkage-specific derivatization of minor amounts of a potential $\alpha 2,6$-sialyllactitol contaminant may likewise contribute to this signal [22]. The relative abundance of the $\mathrm{Me}_{2} \mathrm{NH}$ amidated by-product decreased to $0.9 \pm 0.1 \%$ and $1.5 \% \pm$ $0.1 \%$ after $\mathrm{NH}_{3}$ amidation or $\mathrm{MeNH}_{2}$ amidation, respectively. The minor relative abundance deviations between the lactone, ammonia amide and methylamide forms may be caused by differences in ionization efficiency of the reaction products [32] and/or from subtle response factor differences of the MALDI-TOF-MS detector [33].

Next, the alkaline $\mathrm{NH}_{3}$ or $\mathrm{MeNH}_{2}$ amidation conditions used in the second step of common protocols [16, 23, 25] described above were directly applied on the untreated $\alpha 2,3$ linked standard (Supplementary Table 6). This resulted in only a small proportion of the $\alpha 2,3$-sialyllactitol to be amidated using either EE or DMA reagent in combination with $\mathrm{NH}_{4} \mathrm{OH}$ or $\mathrm{MeNH}_{2}$. The highest conversion was observed for DMA + AA, resulting in $12.0 \pm 1.6 \% \mathrm{NH}_{3}$ amidated product (Fig. 3 "Direct") (Supplementary Fig. 4 and 5) (Supplementary
Table 6). This indicates that prior lactone formation is essential to complete amidation of $\alpha 2,3$-linked sialic acids under the described conditions using EDC and HOBt (or analogues thereof). The alkaline reaction conditions did also not result in any modification of the $\alpha 2,6$-sialyllactitol standards run in parallel, which is in accordance with the acidic $\mathrm{pH}$ optimum of EDC reactivity [6, 34] (Supplementary Fig. 6 and 7).

To further confirm the lactone dependence of high-pH $\alpha 2,3$-linked sialic acid amidation, reactions identical to the native ones were performed, but excluding the presence of EDC and HOBt. These conditions were not supposed to induce lactone formation and indeed resulted in only trace amount of the lactonized $\alpha 2,3$-sialyllactitol standard (Fig. 3 "Control") (Supplementary Fig. 4 and 5) (Supplementary Table 6), which in addition rather originated from the reducing step (Supplementary Fig. 2). Correspondingly low amidation rates were detected, independently from the chosen solvent and nucleophile (Fig. 3) (Supplementary Fig. 4 and 5) (Supplementary Table 6). In addition, no modifications were observed on the $\alpha 2,6$-linked sialyllactitol controls (Supplementary Fig. 6 and 7).

From these results one can conclude that under common one-pot linkage-specific sialic acid derivatization conditions, prior lactone formation is a prerequisite for complete, linkage- 


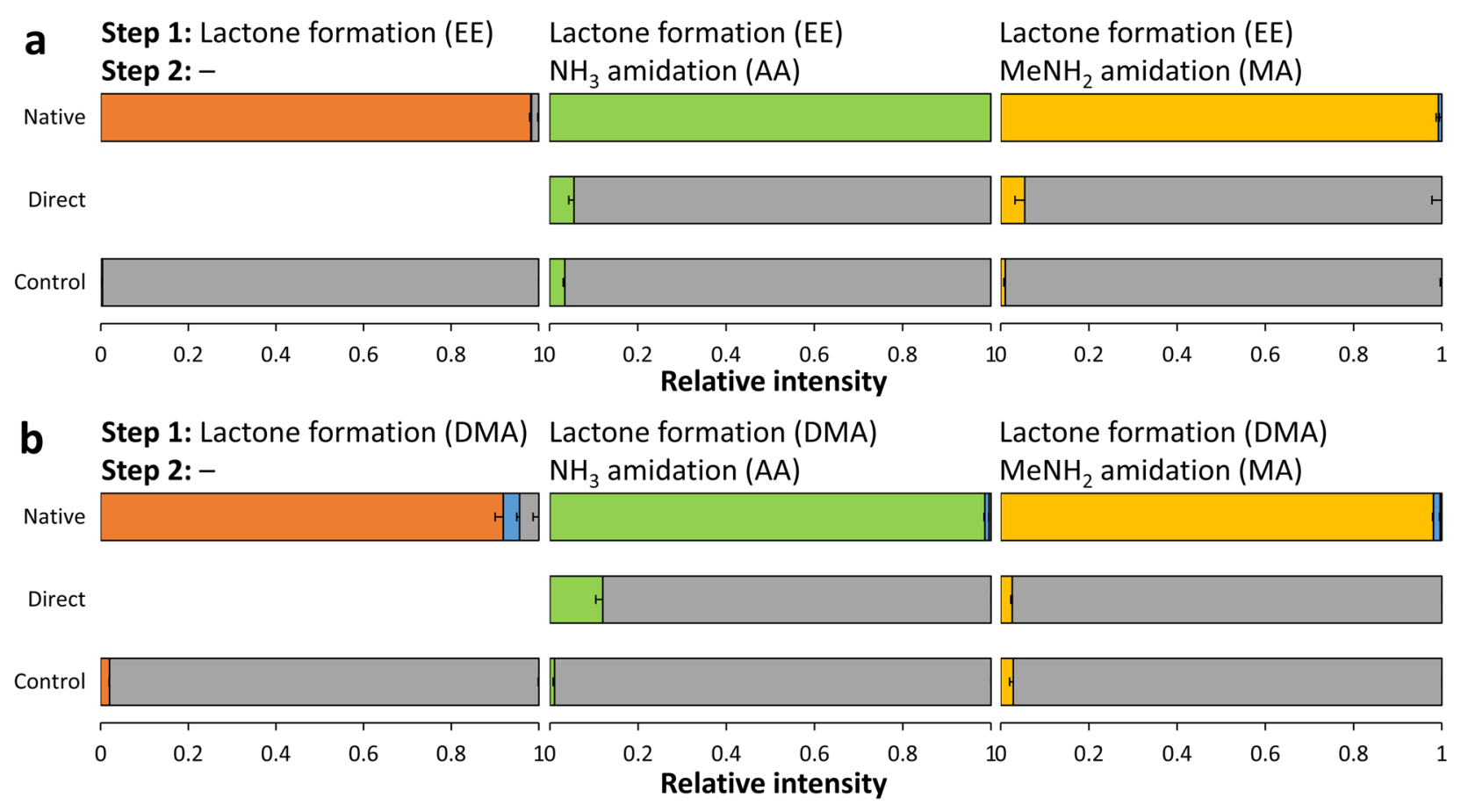

\section{Lactone $\square$ Unmodified $\square$ Ammonia amide}

Fig. 3 Bar graphs illustrating the role of the lactone intermediate on the $\mathrm{NH}_{3}$ amidation and $\mathrm{MeNH}_{2}$ amidation step in EtOH (a) or DMSO (b) using an $\alpha 2,3$-sialyllactitol standard. Native reaction conditions refer to the application of either EE or DMA reagent in the first step of the reaction and the addition of $\mathrm{NH}_{4} \mathrm{OH}$ or $\mathrm{MeNH}_{2}$ in the second step. The direct conditions mimic the $\mathrm{NH}_{3}$ amidation or $\mathrm{MeNH}_{2}$ amidation step by

specific amidation of $\alpha 2,3$-linked sialyllactitol. The reaction proceeds mainly via direct aminolysis and independently from EDC and HOBt. At the same time, free carboxylic acids of $\alpha 2,3$-sialyllactitol react to a minor extent with $\mathrm{NH}_{3}$ and $\mathrm{MeNH}_{2}$, exclusively in the presence of EDC and HOBt. Thus, both proposed mechanisms potentially co-occur, with direct aminolysis being essential for complete amidation of the $\alpha 2,3$-linked sialic acids (Fig. 3). In view of these results, the conventional incubation time in the second step of such reactions $[16,17,19,23,25]$ may significantly be shortened, as direct aminolysis has been described as an instantaneous reaction [24]. Furthermore, for protocols where the lactonized species are purified under mild conditions at neutral $\mathrm{pH}$ prior to treatment with a second nucleophile [16], the re-addition of carboxylic acid activators is not required. While, the direct aminolysis was here demonstrated for $\alpha 2,3$ linked sialic acids only, the proposed mechanisms are also likely to be true for $\alpha 2,8$ - and $\alpha 2,9$-linked sialic acids, which are as well prone to form lactones [35]. Lactone mediated amidation was shown for $\alpha 2,8$-linked sialic acids on glycosphingolipid glycans previously [24].

For further investigations, exclusively the DMA + MA conditions were used. As opposed to the ethyl esterified $\alpha 2,6$-sialyllactitol the $\mathrm{Me}_{2} \mathrm{NH}$ amidated product was not affected by alkaline cleavage using $\mathrm{NH}_{4} \mathrm{OH}$ or $\mathrm{MeNH}_{2}$ the immediate addition of $\mathrm{NH}_{4} \mathrm{OH}$ or $\mathrm{MeNH}_{2}$ in the first step. The control conditions refer to the use of control-reagents without EDC and $\mathrm{HOBt}$ following the two-step reaction. The average and SDs for triplicate measurements are shown as stacked bars and error bars, respectively. The $\mathrm{pH}$ of the conditions was evaluated in triplicate using narrow range $\mathrm{pH}$ indicator strips (Supplementary Table 1)

(Supplementary Fig. 6-8) (Supplementary Table 6). Furthermore, the use of $\mathrm{MeNH}_{2}$ and not $\mathrm{NH}_{4} \mathrm{OH}$ for the second reaction step resulted in easier interpretable spectra by preventing the overlap between unmodified and $\mathrm{NH}_{3}$ amidated species (Supplementary Fig. 4).

\section{The role of the lactone intermediate in the amidation of complex $\mathrm{N}$-glycans}

To validate if our findings hold true for more complex sialylated glycans, $\alpha 2,3$-sialylated $N$-glycans released from rhEPO were subjected to linkage-specific sialic acid derivatization using the DMA + MA approach. The most abundant glycans were singly fucosylated, highly sialylated, partially $O$-acetylated complex-type $\mathrm{N}$-glycans with a varying number of LacNAc units, as described previously [33] (Supplementary Fig. 9). In the first step of the DMA + MA reaction, lactonization was observed for the sialic acids on the mono-, di-, and trisialylated species H5N4F1S1, H5N4F1S2 and H6N5F1S3 (Fig. 4) (Supplementary Table 7). In the second step of native reaction conditions, fully $\mathrm{MeNH}_{2}$ amidated analytes were observed for mono- and disialylated structures with only unquantifiable traces $(\mathrm{S} / N<3)$ of misconversion due to $\mathrm{Me}_{2} \mathrm{NH}$ amidation (Fig. 4a, b) (Supplementary Table 7). On the other hand, $78.5 \pm 0.8 \%$ of the trisialylated 


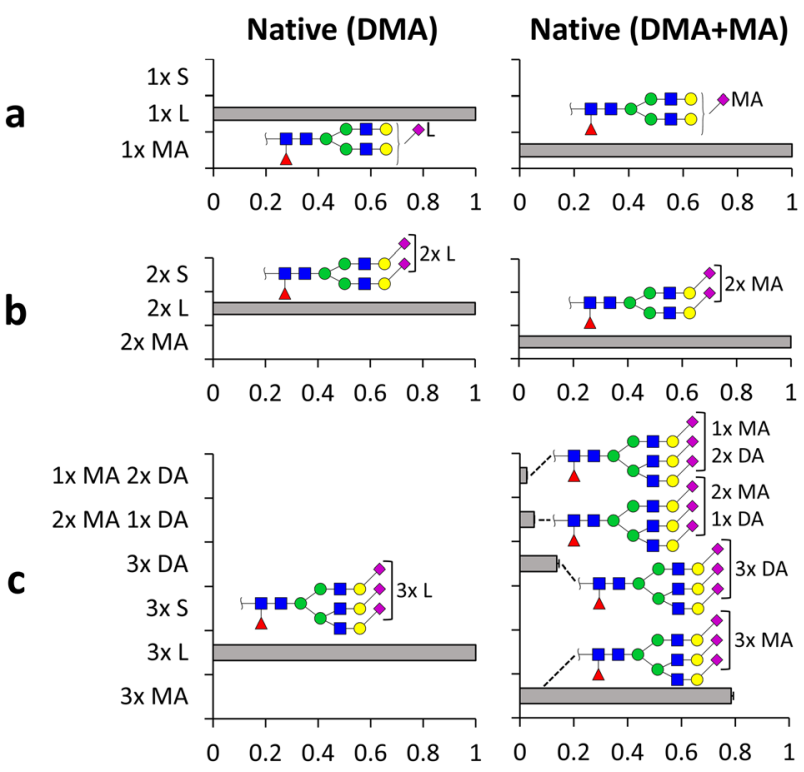

Fig. 4 Reaction products observed for $\alpha 2,3$-linked $\mathrm{N}$-glycans from rhEPO under different conditions. (a) H5N4F1S1; (b) H5N4F1S2 and (c) H6N5F1S3 are the gross glycan compositions evaluated. Abbreviations indicate $\mathrm{H}$ (hexose); $\mathrm{N}$ ( $\mathrm{N}$-acetylgalactosamine); $\mathrm{F}$ (fucose); $\mathrm{S}$ ( $N$-acetylneuraminic acid). Symbols indicate the monosaccharide residues mannose (green circle), galactose (yellow circle), $\mathrm{N}$ acetylgalactosamine (blue square), fucose (red triangle), and $\mathrm{N}$ -

analytes underwent $\mathrm{MeNH}_{2}$ amidation (Fig. 4c) (Supplementary Table 7). Of note, this substrate was in addition found in singly, doubly, as well triply $\mathrm{Me}_{2} \mathrm{NH}$ amidated form, induced by the remaining $\mathrm{Me}_{2} \mathrm{NH}$ from the first step (Fig. 4c) (Supplementary Table 7), as previously reported [16].

When subjected to direct high $\mathrm{pH} \mathrm{MeNH}_{2}$ amidation conditions, none of the analytes were $\mathrm{MeNH}_{2}$ amidated. Similarly as found for the sialyllactitol standards, this indicates that prior lactone formation is essential for the amidation of $\alpha 2,3$-linked sialic acids under the reported conditions. Likewise, with the application of control-reagents deprived from EDC and $\mathrm{HOBt}$, all analytes remained unmodified, indicating that no carboxyl group modification takes place without prior carboxyl activation (Fig. 4). Of note, the instability of unmodified sialic acids lead to in-source desialylation during MALDI-MS and $\mathrm{N}$-glycan acetylation is partly lost under the described reaction conditions, hence causing an underestimation of these species (Supplementary Fig. 9).

To ensure the sialic acid linkage-specificity of the reaction conditions, the described experimental conditions were in addition performed using released glycans deriving from an IgG standard (Supplementary Fig. 10), for which sialylated species have been reported to be almost exclusively $\alpha 2,6$ linked [36]. Under DMA + MA conditions, the detected mono- and disialylated species were entirely $\mathrm{Me}_{2} \mathrm{NH}$ amidated (Supplementary Fig. 11) (Supplementary

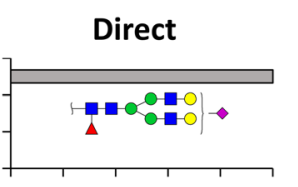

Control (DMA)

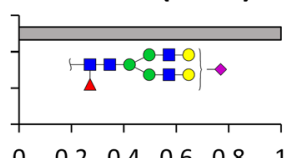

Control (DMA+MA)
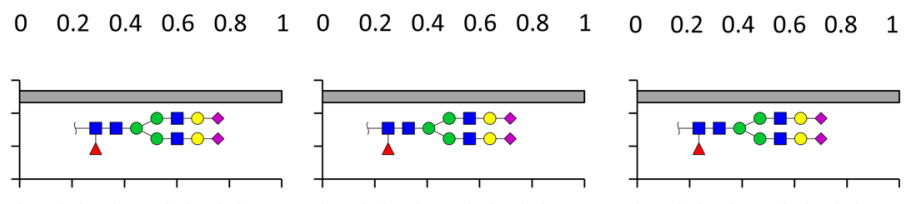

$\begin{array}{llllll}0 & 0.2 & 0.4 & 0.6 & 0.8 & 1\end{array}$
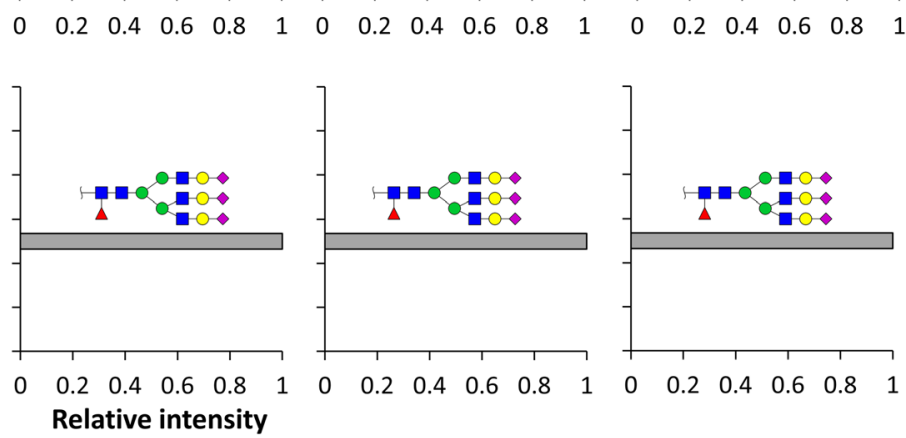

acetylneuraminic acid (purple diamond). In case of derivatized sialic acids, an $\alpha 2,3$-linkage is depicted with a left angle, with lactonization indicated by $\mathrm{L}, \mathrm{MeNH}_{2}$ amidation by $\mathrm{MA}$, and $\mathrm{Me}_{2} \mathrm{NH}$ amidation by DA next to the sialic acid residue. Non-derivatized $\alpha 2,3$-sialic acids are depicted without an angle. The average and SDs for triplicate measurements are shown as bars and error bars, respectively (Supplementary Table 7)

Table 7). The obtained glycosylation profiles and relative sialic acid abundancies for IgG as well as rhEPO were highly similar to what was previously reported for these glycoproteins $[33,36]$.

\section{Structural characterization of the lactone}

While NMR spectroscopy has previously been utilized to characterize sialyllactones of varying origin, the reaction products obtained with carboxylic acid activators commonly used in sialic acid derivatization, especially in the presence of a catalyst and a nucleophile, have not yet been elucidated in a similar manner [37, 38]. To address this, we first recorded a $1 \mathrm{D}{ }^{1} \mathrm{H}$ NMR spectrum of $\alpha 2,3-\mathrm{SL}$ dissolved in $\mathrm{D}_{2} \mathrm{O}$ as a reference spectrum (Fig. 5). Second, lactonization of $\alpha 2,3-$ SL was performed under DMA conditions as described above, and the products were analyzed by $1 \mathrm{D}{ }^{1} \mathrm{H}$ NMR. Not all peaks of $\alpha 2,3-\mathrm{SL}$ were properly resolved in the 1D NMR spectrum, due to the strong coupling effects and resulting overlap in the region between 3.55 and $4.01 \mathrm{ppm}$. However, the peaks that were resolved allowed the identification of the lactone and to follow the hydrolysis product over time (Fig. 5). Exclusively the $\mathrm{C} 2$ lactone was detected, indicated by the diagnostic ppms (Supplementary Table 8). As determined from the peak integrals, a significant amount (64\%) of unmodified $\alpha 2,3$-SL was detected already at the first measurement timepoint. This was likely a result of sample hydrolysis, as a measurement time of 
Fig. $51 \mathrm{D}^{1} \mathrm{H}$ NMR spectra of the reference $\alpha 2,3$-sialyllactose standard and its $\mathrm{C} 2$ lactone derivative. Overlaid spectra of the reference $\alpha 2,3$-sialyllactose standard (green), the same standard after subjected to lactone formation (blue), and the lactonized standard $16 \mathrm{~h}$ after the first measurement (orange). The lactone peaks are marked with an asterisk. Contaminant peaks of dimethylamine and ACN originate from cotton HILIC SPE washing steps

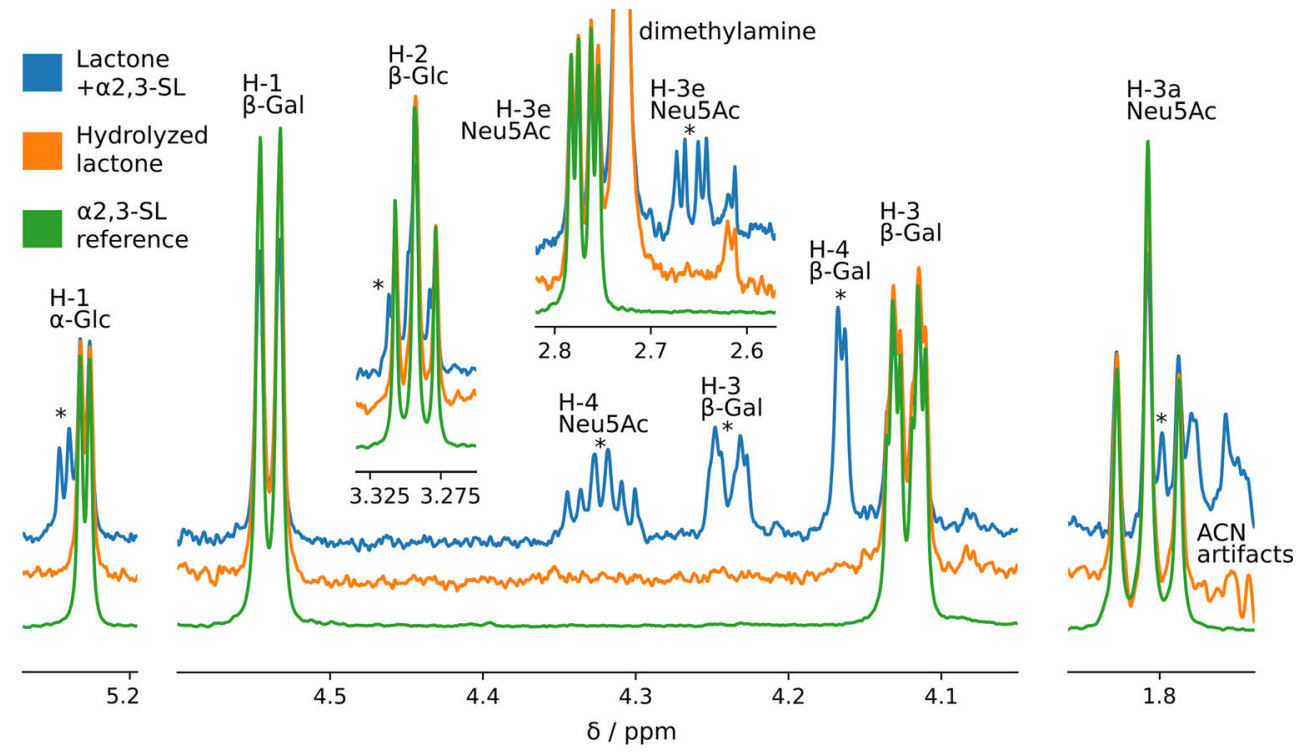

approximately $1 \mathrm{~h}$ was required to obtain sufficient sensitivity. When remeasuring the sample after $16 \mathrm{~h}$ of storage at $300 \mathrm{~K}$, only unmodified $\alpha 2,3$-SL was detectable, indicating completed lactone hydrolysis. Of note, the $\mathrm{C} 4$ lactone was not detected, either due to its low abundance or rather quick hydrolysis during analysis. However, rapid hydrolysis of the $\mathrm{C} 4$ lactone is unlikely, as it has been reported to be more resistant to hydrolysis in an $\alpha 2,3-\mathrm{SL}$ structure, as opposed to the $\mathrm{C} 2$ lactone [39]. The results are in alignment with previous reports on glacial acetic acid induced lactonization of $\alpha 2,3$-SL, showing that this structure mainly results in a $\mathrm{C} 2$ lactone [39]. Likewise, ganglioside GM3 lactonization was found to result in a C2 lactone [40]. In contrast, lactonization of a synthetic sialyl T benzyl glycoside resulted in $\mathrm{C} 2$ and $\mathrm{C} 4$ lactones in 3:2 ratio and a synthetic MUC1 glycopeptide with a sialyl T glycan moiety mainly yielded $\mathrm{C} 4$ lactones following glacial acetic acid treatment [41]. Literature suggests that the lactone variants formed and their distinct hydrolytic stability mainly depends on the rest of the glycoconjugate [39, 41]. From the current data it remains unclear whether a potential $\mathrm{C} 4$ lactone can be amidated via direct aminolysis with the same efficiency as the $\mathrm{C} 2$ lactone. Therefore, the extrapolation of the here presented results on sialyllactose to more complex glycoconjugates is not straightforward and requires the investigation of a wider variety of pure glycoconjugate standards by NMR instead.

\section{Conclusions}

In this report, we investigated the lactone intermediate and its role in the amidation of $\alpha 2,3$-linked sialic acids in one-pot linkage-specific sialic acid derivatization workflows. We demonstrated that the amidation of $\alpha 2,3$-linked sialic acids occurs predominantly by direct aminolysis, and to a minor and likely negligible extent via the reaction of the free carboxyl group in the presence of a carboxylic acid activator and catalyst. We believe this report resolves the former ambiguity around the structure and role of the lactone intermediate in one-pot, in-solution, linkage-specific sialic acid derivatization reactions. As a consequence, this type of protocols has the potential to be significantly shortened and the addition of carboxylic acid activators in the second step of these reactions can be omitted.

Supplementary Information The online version contains supplementary material available at https://doi.org/10.1007/s10719-020-09971-7.

Acknowledgments We thank Simone Nicolardi for his help with the MALDI-FT-ICR-MS/MS analysis.

Authors' contributions T. P.: Methodology, data curation, formal analysis, validation, investigation, visualization, writing - original draft preparation. A. V.: Investigation, visualization. M. W.: Supervision, writing editing, funding acquisition. N. de H.: Conceptualization, methodology, supervision, writing - editing.

All authors have given approval to the final version of the manuscript.

Funding This project has received funding from the European Commission's Horizon2020 research and innovation programme for H2020-MSCA-ITN IMforFUTURE, under grant agreement number 721815 .

Data availability The datasets generated during and/or analyzed during the current study are available from the corresponding author on reasonable request.

\section{Compliance with ethical standards}

Conflict of interest M. Wuhrer is inventor on several patents on derivatizing sialic acids for high-throughput glycosylation profiling. The authors declare that they have no conflicts of interest. 
Ethical approval This article does not contain any studies with human participants or animals performed by any of the authors.

Open Access This article is licensed under a Creative Commons Attribution 4.0 International License, which permits use, sharing, adaptation, distribution and reproduction in any medium or format, as long as you give appropriate credit to the original author(s) and the source, provide a link to the Creative Commons licence, and indicate if changes were made. The images or other third party material in this article are included in the article's Creative Commons licence, unless indicated otherwise in a credit line to the material. If material is not included in the article's Creative Commons licence and your intended use is not permitted by statutory regulation or exceeds the permitted use, you will need to obtain permission directly from the copyright holder. To view a copy of this licence, visit http://creativecommons.org/licenses/by/4.0/.

\section{References}

1. Varki, A.: Biological roles of glycans. Glycobiology. 27(1), 3-49 (2017). https://doi.org/10.1093/glycob/cww086

2. Varki, A.: Biological roles of oligosaccharides: all of the theories are correct. Glycobiology. 3(2), 97-130 (1993). https://doi.org/10. 1093/glycob/3.2.97

3. Ohtsubo, K., Marth, J.D.: Glycosylation in cellular mechanisms of health and disease. Cell. 126(5), 855-867 (2006). https://doi.org/ 10.1016/j.cell.2006.08.019

4. Varki, A.: Glycan-based interactions involving vertebrate sialicacid-recognizing proteins. Nature. 446(7139), 1023-1029 (2007). https://doi.org/10.1038/nature05816

5. Schauer, R., Kamerling, J.P.: Exploration of the Sialic acid world. Adv. Carbohydr. Chem. Biochem. 75, 1-213 (2018). https://doi. org/10.1016/bs.accb.2018.09.001

6. Yang, S., Jankowska, E., Kosikova, M., Xie, H., Cipollo, J.: Solidphase chemical modification for Sialic acid linkage analysis: application to glycoproteins of host cells used in influenza virus propagation. Anal. Chem. 89(17), 9508-9517 (2017). https://doi.org/10. 1021/acs.analchem.7b02514

7. Air, G.M.: Influenza virus-glycan interactions. Curr Opin Virol. 7, 128-133 (2014). https://doi.org/10.1016/j.coviro.2014.06.004

8. Ilver, D., Johansson, P., Miller-Podraza, H., Nyholm, P.-G., Teneberg, S., Karlsson, K.-A.: Bacterium-Host ProteinCarbohydrate Interactions. In: Methods in Enzymology, vol. 363. pp. 134-157. Academic Press, (2003)

9. Morell, A.G.G.G., Scheinberg, I.E., Hickman, J., Ashwell, G.: The Role of Sialic Acid in Determining the Survival of Glycoproteins in the Circulation. J. Biol. Chem. 246(10), 1461-1467 (1971)

10. Tyrrell, D., James, P., Rao, N., Foxall, C., Abbas, S., Dasgupta, F., Nashed, M., Hasegawa, A., Kiso, M., Asa, D., et al.: Structural requirements for the carbohydrate ligand of E-selectin. Proc. Natl. Acad. Sci. U. S. A. 88(22), 10372-10376 (1991). https://doi.org/10. 1073/pnas.88.22.10372

11. Oswald, D.M., Jones, M.B., Cobb, B.A.: Modulation of hepatocyte sialylation drives spontaneous fatty liver disease and inflammation. Glycobiology. 30, 346-359 (2019). https://doi.org/10.1093/glycob/ cwz096

12. Zhang, Z., Wuhrer, M., Holst, S.: Serum sialylation changes in cancer. Glycoconj. J. 35(2), 139-160 (2018). https://doi.org/10. 1007/s10719-018-9820-0

13. Pearce, O.M., Laubli, H.: Sialic acids in cancer biology and immunity. Glycobiology. 26(2), 111-128 (2016). https://doi.org/10. 1093/glycob/cwv097

14. Reiding, K.R., Blank, D., Kuijper, D.M., Deelder, A.M., Wuhrer, M.: High-throughput profiling of protein N-glycosylation by
MALDI-TOF-MS employing linkage-specific sialic acid esterification. Anal. Chem. 86(12), 5784-5793 (2014). https://doi.org/10. 1021/ac500335t

15. Wheeler, S.F., Domann, P., Harvey, D.J.: Derivatization of sialic acids for stabilization in matrix-assisted laser desorption/ionization mass spectrometry and concomitant differentiation of alpha $(2 \rightarrow$ $3)-$ and alpha( $2 \rightarrow 6)$-isomers. Rapid Commun. Mass Spectrom. 23(2), 303-312 (2009). https://doi.org/10.1002/rcm.3867

16. Suzuki, N., Abe, T., Natsuka, S.: Quantitative LC-MS and MS/MS analysis of sialylated glycans modified by linkage-specific alkylamidation. Anal. Biochem. 567, 117-127 (2019). https://doi. org/10.1016/j.ab.2018.11.014

17. Nishikaze, T., Tsumoto, H., Sekiya, S., Iwamoto, S., Miura, Y., Tanaka, K.: Differentiation of Sialyl linkage isomers by one-pot Sialic acid Derivatization for mass spectrometry-based glycan profiling. Anal. Chem. 89(4), 2353-2360 (2017). https://doi.org/10. 1021/acs.analchem.6b04150

18. Liu, X., Qiu, H., Lee, R.K., Chen, W., Li, J.: Methylamidation for sialoglycomics by MALDI-MS: a facile derivatization strategy for both alpha2,3- and alpha2,6-linked sialic acids. Anal. Chem. 82(19), 8300-8306 (2010). https://doi.org/10.1021/ac101831t

19. Li, H., Gao, W., Feng, X., Liu, B.F., Liu, X.: MALDI-MS analysis of sialylated $\mathrm{N}$-glycan linkage isomers using solid-phase two step derivatization method. Anal. Chim. Acta. 924, 77-85 (2016). https://doi.org/10.1016/j.aca.2016.04.023

20. de Haan, N., Reiding, K.R., Haberger, M., Reusch, D., Falck, D., Wuhrer, M.: Linkage-specific sialic acid derivatization for MALDITOF-MS profiling of IgG glycopeptides. Anal. Chem. 87(16), 8284-8291 (2015). https://doi.org/10.1021/acs.analchem.5b02426

21. Vreeker, G.C.M., Nicolardi, S., Bladergroen, M.R., van der Plas, C.J., Mesker, W.E., Tollenaar, R.A.E.M., van der Burgt, Y.E.M., Wuhrer, M.: Automated plasma Glycomics with linkage-specific Sialic acid esterification and ultrahigh resolution MS. Anal. Chem. 90(20), 11955-11961 (2018). https://doi.org/10.1021/acs. analchem.8b02391

22. Pongracz, T., Wuhrer, M., de Haan, N.: Expanding the Reaction Space of Linkage-Specific Sialic Acid Derivatization. Molecules 24(19) (2019). https://doi.org/10.3390/molecules24193617

23. Holst, S., Heijs, B., de Haan, N., van Zeijl, R.J., Briaire-de Bruijn, I.H., van Pelt, G.W., Mehta, A.S., Angel, P.M., Mesker, W.E., Tollenaar, R.A., Drake, R.R., Bovee, J.V., McDonnell, L.A., Wuhrer, M.: Linkage-specific in situ Sialic acid Derivatization for $\mathrm{N}$-glycan mass spectrometry imaging of formalin-fixed paraffinembedded tissues. Anal. Chem. 88(11), 5904-5913 (2016). https://doi.org/10.1021/acs.analchem.6b00819

24. Hanamatsu, H., Nishikaze, T., Miura, N., Piao, J., Okada, K., Sekiya, S., Iwamoto, S., Sakamoto, N., Tanaka, K., Furukawa, J.I.: Sialic acid linkage specific Derivatization of Glycosphingolipid Glycans by ring-opening Aminolysis of lactones. Anal. Chem. 90(22), 13193-13199 (2018). https://doi.org/ 10.1021/acs.analchem.8b02775

25. Lageveen-Kammeijer, G.S.M., de Haan, N., Mohaupt, P., Wagt, S., Filius, M., Nouta, J., Falck, D., Wuhrer, M.: Highly sensitive CEESI-MS analysis of N-glycans from complex biological samples. Nat. Commun. 10(1), 2137 (2019). https://doi.org/10.1038/s41467019-09910-7

26. de Haan, N., Yang, S., Cipollo, J., Wuhrer, M.: Glycomics studies using sialic acid derivatization and mass spectrometry. Nature Rev. Chem. 4, 229-242 (2020). https://doi.org/10.1038/s41570-0200174-3

27. Nishikaze, T.: Sialic acid derivatization for glycan analysis by mass spectrometry. Proc Japan Acad, Series B. 95(9), 523-537 (2019). https://doi.org/10.2183/pjab.95.036

28. Jensen, P.H., Karlsson, N.G., Kolarich, D., Packer, N.H.: Structural analysis of $\mathrm{N}$ - and O-glycans released from glycoproteins. Nat. 
Protoc. 7(7), 1299-1310 (2012). https://doi.org/10.1038/nprot. 2012.063

29. Rebello, O.D., Nicolardi, S., Lageveen-Kammeijer, G.S.M., Nouta, J., Gardner, R.A., Mesker, W.E., Tollenaar, R.A.E.M., Spencer, D.I.R., Wuhrer, M., Falck, D.: A Matrix-Assisted Laser Desorption/Ionization-Mass Spectrometry Assay for the Relative Quantitation of Antennary Fucosylated N-Glycans in Human Plasma. Frontiers in Chemistry 8 (2020). https://doi.org/ 10.3389/fchem. 2020.00138

30. Selman, M.H.J., Hemayatkar, M., Deelder, A.M., Wuhrer, M.: Cotton HILIC SPE microtips for microscale purification and enrichment of Glycans and Glycopeptides. Anal. Chem. 83(7), 24922499 (2011). https://doi.org/10.1021/ac1027116

31. Jansen, B.C., Reiding, K.R., Bondt, A., Hipgrave Ederveen, A.L., Palmblad, M., Falck, D., Wuhrer, M.: MassyTools: a highthroughput targeted data processing tool for relative quantitation and quality control developed for Glycomic and Glycoproteomic MALDI-MS. J. Proteome Res. 14(12), 5088-5098 (2015). https:// doi.org/10.1021/acs.jproteome.5b00658

32. Peng, Y., Wang, L., Zhang, Y., Bao, H., Lu, H.: Stable isotope sequential Derivatization for linkage-specific analysis of Sialylated N-glycan isomers by MS. Anal. Chem. 91(24), 1599316001 (2019). https://doi.org/10.1021/acs.analchem.9b04727

33. Falck, D., Haberger, M., Plomp, R., Hook, M., Bulau, P., Wuhrer, M., Reusch, D.: Affinity purification of erythropoietin from cell culture supernatant combined with MALDI-TOF-MS analysis of erythropoietin N-glycosylation. Sci. Rep. 7(1), 5324 (2017). https://doi.org/10.1038/s41598-017-05641-1

34. Yang, S., Wu, W.W., Shen, R.F., Bern, M., Cipollo, J.: Identification of Sialic acid linkages on intact Glycopeptides via differential chemical modification using IntactGIG-HILIC. J. Am. Soc. Mass Spectrom. 29(6), 1273-1283 (2018). https://doi.org/10. 1007/s13361-018-1931-0

35. Galuska, S.P., Geyer, R., Muhlenhoff, M., Geyer, H.: Characterization of oligo- and polysialic acids by MALDI-TOF-
MS. Anal. Chem. 79(18), 7161-7169 (2007). https://doi.org/10. 1021/ac0712446

36. Anthony, R.M., Nimmerjahn, F., Ashline, D.J., Reinhold, V.N., Paulson, J.C., Ravetch, J.V.: Recapitulation of IVIG antiinflammatory activity with a recombinant IgG fc. Science. 320(5874), 373-376 (2008). https://doi.org/10.1126/science. 1154315

37. Sonnino, S., Kirschner, G., Fronza, G., Egge, H., Ghidoni, R., Acquotti, D., Tettamanti, G.: Synthesis of GM1-Ganglioside inner Ester. Glycoconj. J. 2(3-4), 343-354 (2005). https://doi.org/10. 1007/bf03402223

38. Fronza, G., Kirschner, G., Acquotti, D., Bassi, R., Tagliavacca, L., Sonnino, S.: Synthesis and structural characterization of the dilactone derivative of GDla ganglioside. Carbohydr. Res. 182(1), 31-40 (1988). https://doi.org/10.1016/0008-6215(88) 84089-8

39. Nakamura, T., Bubb, W.A., Saito, T., Arai, I., Urashima, T.: An NMR study of the lactonization of $\alpha$-N-acetylneuraminyl-(2 $\rightarrow 3)$ lactose. Carbohydr. Res. 329(2), 471-476 (2000). https://doi.org/ 10.1016/s0008-6215(00)00202-0

40. Yu, R.K., Koerner, T.A., Ando, S., Yohe, H.C., Prestegard, J.H.: High-resolution proton NMR studies of gangliosides. III. Elucidation of the structure of ganglioside GM3 lactone. J Biochem. 98(5), 1367-1373 (1985). https://doi.org/10.1093/ oxfordjournals.jbchem.a135404

41. Pudelko, M., Lindgren, A., Tengel, T., Reis, C.A., Elofsson, M., Kihlberg, J.: Formation of lactones from sialylated MUC1 glycopeptides. Org Biomol Chem. 4(4), 713-720 (2006). https://doi.org/ $10.1039 / \mathrm{b} 514918 \mathrm{e}$

Publisher's note Springer Nature remains neutral with regard to jurisdictional claims in published maps and institutional affiliations. 